\title{
Anatomic aspects of the atrioventricular junction influencing radiofrequency Cox maze IV procedures
}

\author{
Manuel Castellá, MD, PhD, ${ }^{a}, *$ Antonio García-Valentín, MD, ${ }^{a}$ Daniel Pereda, MD, ${ }^{a}$ Andrea Colli, MD, ${ }^{a}$ \\ Antonio Martinez, MD, ${ }^{\mathrm{b}}$ Daniel Martinez, MD, ${ }^{\mathrm{b}}$ José Ramirez, MD, ${ }^{\mathrm{b}}$ and Jaime Mulet, MD, $\mathrm{PhD}^{\mathrm{a}}$
}

From the Department of Cardiovascular Surgery, Institut del Tòrax, ${ }^{\mathrm{a}}$ and the Department of Pathology, ${ }^{\mathrm{b}}$ Hospital Clínic, University of Barcelona, Barcelona, Spain.

* Dr Castellá is a Consultant to AtriCure, Inc.

Received for publication Dec 13, 2007; revisions received Feb 12, 2008; accepted for publication March 13, 2008

Address for reprints: Manuel Castellá, MD $\mathrm{PhD}$, Department of Cardiovascular Surgery, Hospital Clínic, C/Villarroel 17008036 Barcelona, Spain (E-mail: mcaste@ clinic.ub.es).

J Thorac Cardiovasc Surg 2008;136:419-23 $0022-5223 / \$ 34.00$

Copyright $(2008$ by The American Association for Thoracic Surgery

doi:10.1016/j.jtcvs.2008.03.049
Objective: This study analyzes the anatomic structure of the mitral and tricuspid annuli, their relationship with the coronary arteries and veins, and how this anatomic distribution may affect atrial ablation with bipolar radiofrequency clamps, the only technology that ensures transmurality.

Methods: Nine explanted fresh human hearts were studied, two of them with left coronary dominance. Two types of bipolar radiofrequency clamps were positioned to reach the mitral and tricuspid annuli, and relationships within the atrioventricular junction were analyzed, including coronary sinus and coronary arteries.

Results: In all hearts studied, the coronary arteries and veins within the adipose tissue of the right or left atrioventricular groove lay in the atrial side, 3 to $18 \mathrm{~mm}$ away from the mitral or tricuspid annuli. When the bipolar radiofrequency clamp was closed toward the mitral annulus, the coronary sinus was always included between the jaws, and in left coronary-dominant hearts, the circumflex artery was also included. Nevertheless, the clamp never reached the annulus owing to the increase in thickness of the adipose tissue around the groove and the ventricular mass, leaving 5 to $10 \mathrm{~mm}$ of atrial myocardium free from the radiofrequency electrodes. In the right atrium, clamp placement toward the tricuspid annulus excluding the right coronary left 8 to $18 \mathrm{~mm}$ of atrial muscle free from the bipolar electrodes.

Conclusions: Bipolar radiofrequency clamps are not sufficient to complete a Cox maze IV procedure. Moreover, they may compromise coronary arteries in patients with left coronary dominance. Lines to the atrioventricular annuli need to be completed with the cut-and-sew technique or with alternative monopolar energy devices.

$\mathrm{T}$ The introduction by Dr James Cox in 1991 of the cut-and-sew maze III operation set the gold standard in atrial fibrillation (AF) therapy, with over $90 \%$ cure at long follow-up. ${ }^{1}$ It was based in the theory of multiple re-entrant circuits that needed to be interrupted by conduction block lines, redirecting the electrical impulse into no-exit paths. Despite later knowledge that most of the triggering zones in $\mathrm{AF}$ are focused in or around the pulmonary veins ${ }^{2}$ and that isolation of these zones can eradicate paroxysmal AF, pulmonary vein isolation alone has shown poor results in patients with persistent or long-standing persistent $\mathrm{AF} .{ }^{3}$ Possibly, these patients have additional structural factors resulting from atrial fibrosis and dilatation that may establish permanent macro re-entrant circuits overriding the importance of triggering foci within the pulmonary veins.

Despite the efficacy of the Cox maze III operation, the surgical complexity of the procedure has limited its use and few long series have been reported. Recent introduction of alternative energy devices that can create thermal lesions in a relatively short time and avoid long atrial sutures has impulsed this operation again, with similar conduction block lines as described by Dr James Cox, termed now as the Cox maze IV procedure (Figure 1).

Several alternative energies have been proposed to manage AF. From them, bipolar radiofrequency has been shown to have some advantages. Applied through a 5- to $7-\mathrm{cm}$ clamp, in a short period of seconds it creates a linear lesion. The tissue ablated 


\section{Abbreviations and Acronyms \\ $\mathrm{AF}=$ atrial fibrillation \\ $\mathrm{AV}=$ atrioventricular}

is only that which is included between the jaws of the clamp, so that no possible esophageal or coronary lesion can be produced if correctly applied. Most important, only radiofrequency applied between two gold-plated electrodes has the capability of ensuring transmurality, by measurements of fall of tissue conductance.

A recent report has suggested inferior efficacy of the Cox maze operation when performed with bipolar radiofrequency than with the cut-and-sew technique. ${ }^{4}$ In this study, bipolar ablation was combined with cryolesions to complete the lines toward the mitral and the tricuspid valve annuli, and lack of transmurality arose as a possible factor determining results.

The possibility of reproducing the pattern of lesions of the cut-and-sew maze III with total bipolar radiofrequency may mimic the efficacy of Cox's operation. Our study tried to clarify whether application of bipolar radiofrequency is feasible in the most controversial lines, connecting the mitral and the tricuspid valve annuli.

\section{Material and Methods}

The anatomic study was performed in 8 fresh human hearts from our homograft donor bank (Transplant Services Foundation, Hospital Clínic, Barcelona, Spain) after donor in-life signed consent or donor family's signed authorization and the approval of the Research Committee of the Transplant Services Foundation. Hearts studied were harvested from 6 men and 2 women, age range 25 to 65 years. Studies were performed within 36 hours from donor extraction and after harvesting both the aortic and pulmonary root homografts. Six hearts presented right coronary dominance and two left coronary dominance, which allowed us to study the relationships of the circumflex artery and the mitral annulus, as seen in Figure 2. Distances between the right coronary and tricuspid annulus were also studied in all hearts.

Two bipolar radiofrequency clamps were tested in their position to reach the mitral and tricuspid annuli. Those were the Cardioblate (Medtronic, Inc, Minneapolis, Minn) and AtriCure (AtriCure, Inc, Cincinnati, Ohio) radiofrequency systems. The studies on the mitral annulus were positioning the clamps to include the posterior wall of the left atrium from a standard left atriotomy through the Waterston groove expanding under the inferior vena cava, toward the posterior leaflet of the mitral valve at the level of $\mathrm{P} 2$ and $\mathrm{P} 3$, as presented in Figure 1, $C$. In the tricuspid annulus, we studied the relationships with the right coronary at the level of the anterior leaflet close to the anteroposterior commissure, positioning the clamps from a transverse right atriotomy, as seen in Figure 1, $B$.

We also studied a necropsy heart of a male patient 72 years of age with a history of aortic stenosis and long-standing persistent AF (left atrial diameter $51 \mathrm{~mm}$ ). Preoperative coronary arteriography showed right dominance. The patient died 10 hours after an aortic valve replacement and Cox maze IV procedure by bipolar radiofrequency (AtriCure), following the lesion pattern of Figure 1, $A, B$, and $C$ as we routinely perform for persistent and long-standing persistent AF. The cause of death was not related to the ablation but to sudden bleeding from a very calcified aortotomy after a hypertensive peak at the sixth hour after the operation. After the family signed authorization, a complete necropsy study was performed 8 hours after death. Hematoxylin and eosin staining and complement C9 immunostaining were performed on the ablation lines of the left and right atria. C9 staining has been attributed as one of the earliest and most sensitive markers of necrosis, being able to detect myocardial necrosis 4 to 6 hours after infarction. ${ }^{5}$

\section{Results \\ Left Atrium}

The adipose tissue on the epicardial side of the atrioventricular (AV) junction covers mostly the atrial side of the groove. In all hearts the coronary sinus lay in the epicardial side of the left atrium, being 3 to $10 \mathrm{~mm}$ away from the mitral annulus. In the 2 hearts studied with left coronary dominance, the circumflex artery also lay in the epicardial side of the left atrium, at distances to the mitral annulus that varied from $3 \mathrm{~mm}$ to $10 \mathrm{~mm}$ (Figure 2).

Both Medtronic and AtriCure bipolar clamps were able to be placed in the direction of the mitral annulus. In all cases, what limited how far the clamp could be positioned was the unfeasibility of clamping the ventricular myocardium and the AV adipose tissue that protrudes in the epicardial side. At all times, the maximum amount of atrial tissue clamped was limited by the tip of the epicardial jaw reaching the ventricular myocardial mass. However, the clamp never reached the mitral annulus (Figure 3), leaving from 5 to $10 \mathrm{~mm}$ of atrial muscle out of reach of the bipolar electrodes. This did not limit the device to include the coronary sinus within its jaws in all hearts. Most important, in both hearts in which a left coronary dominance was detected, the circumflex artery was within the bipolar jaws of the clamp.

\section{Right Atrium}

The epicardial side of the tricuspid annulus is covered by adipose tissue that expands to cover the first millimeters of both atrial and ventricular walls. In all hearts studied, both the right coronary artery and vein were in the atrial side of the AV groove and covered by fat. To reach the tricuspid valve annulus at the level of the anterior leaflet with the bipolar radiofrequency clamp was feasible, but it always included both the right coronary artery and vein. If the bipolar clamp was placed up to the epicardial AV adipose tissue or up to the edge of the right coronary artery when identified by dissection, the endocardial jaw of the clamp never reached the tricuspid valve annulus. The distance of atrial myocardium free from thermal ablation, from the tip of the bipolar electrodes to the tricuspid annulus, varied from 8 to $18 \mathrm{~mm}$ (Figure 4).

\section{Postmortem Radiofrequency Ablation Study}

Postmortem examination of the patient who died in the early postoperative period after aortic valve replacement and 

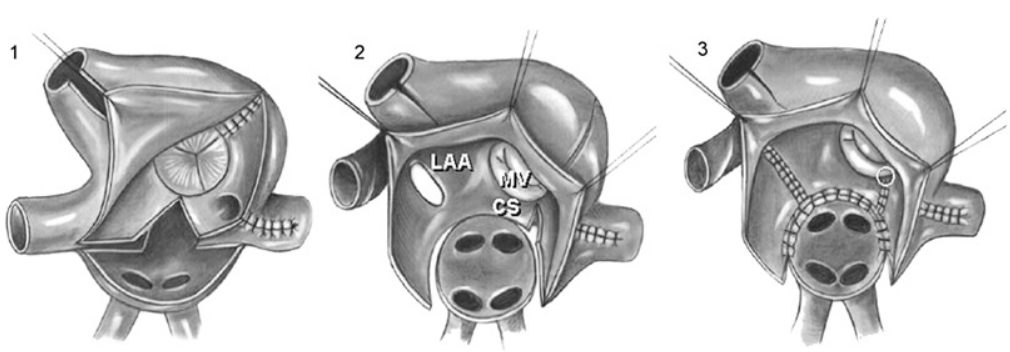

Figure 1. Similar line patterns, different type of lesions: Images 1 to 3 show the lines described by Cox in the cut-and-sew Cox maze III technique. Images A, B, and C show the pattern for a Cox maze IV, combining incisions and bipolar radiofrequency ablation (dotted lines). This lesion set was performed in the ninth heart studied. LAA, Left atrial aappendage; $M V$, mitral valve; $C S$, coronary sinus.
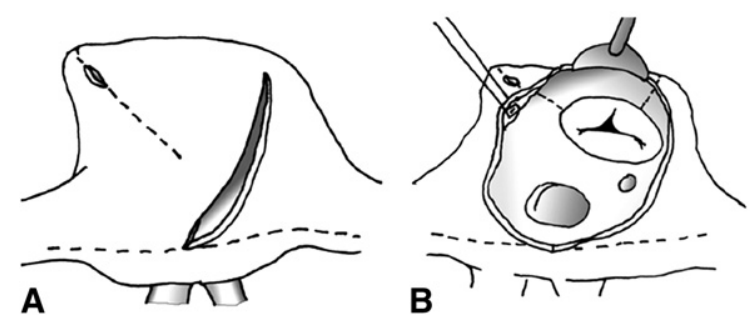

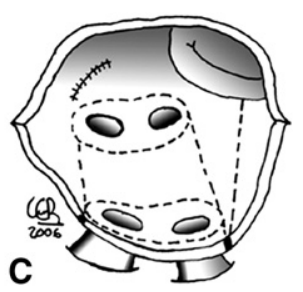

To really reproduce short- and long-term results of the cutand-sew Cox maze operation with alternative energy application, one needs to assure transmural conduction block along the complete set of lines. Bipolar radiofrequency has demonstrated transmurality. However, our study including the two systems with more references in medical literature shows that it is not possible to complete the ablation lines to the mitral or tricuspid valve annuli with actual bipolar radiofrequency technology. Completing the lines from the pulmonary veins to the $\mathrm{AV}$ fibrous tissue still needs to be performed by cut-and-sew technique or by any means of monopolar thermal lesion, either warm or cold, compromising transmurality. At this moment, the combination of bipolar radiofrequency to perform the ablation lines and cryolesions to finish the lines up to the mitral and tricuspid annuli is probably the technique with best known results in Cox maze IV procedures. Nevertheless, this technique has shown less efficacy than the cut-and-sew Cox maze III. ${ }^{4}$ Newly introduced monopolar or transpolar radiofrequency devices still need to prove tissue transmurality and clinical efficacy. Not being able to complete the mitral line may even be detrimental, inasmuch as incomplete lines with narrow paths favor left atrial flutter. ${ }^{8}$ Electrophysiologic studies in patients with atrial flutter after modified radiofrequency maze procedures have
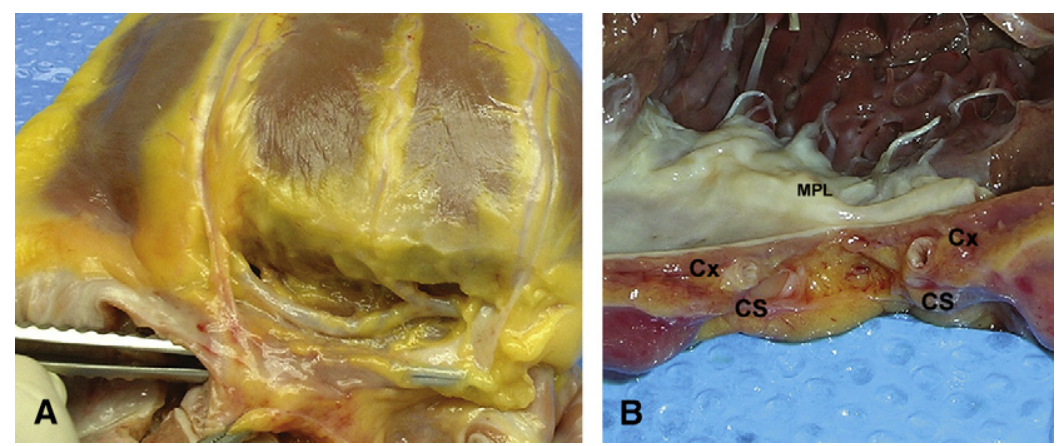

Figure 2. Two examples of left dominant coronary circulation. A, Inferior aspect of the heart, where both the coronary sinus (with pick-ups inside) and the circumflex artery run in the atrial side of the atrioventricular junction. B, A heart with a dominant circumflex artery $\left(C_{X}\right)$ that it is been cut together with the coronary sinus (CS) to reach the mitral annulus. MPL, Mitral posterior leaflet. 


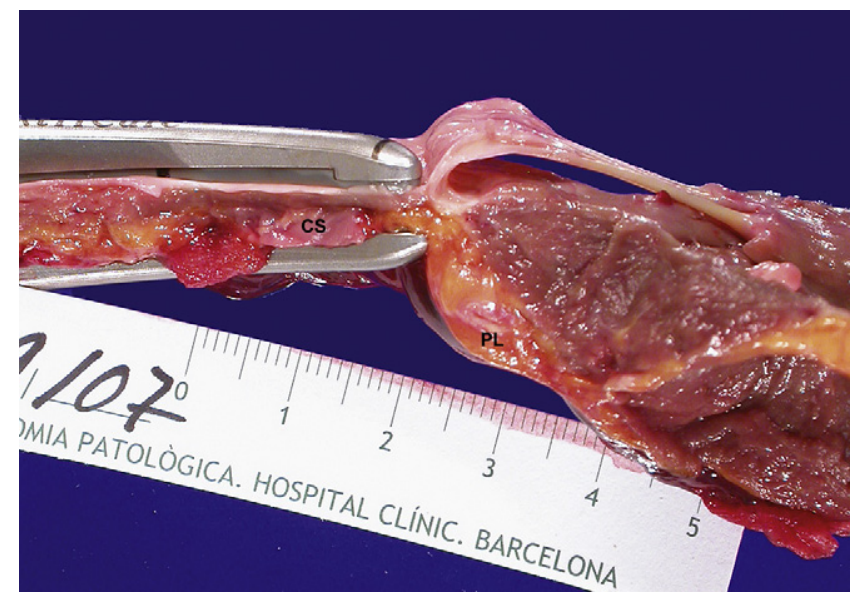

Figure 3. Radiofrequency bipolar clamp positioned to carry out the ablation line from the left auriculotomy to the mitral annulus. The clamp is placed as close as possible to reach the annulus at the level of P2 of the posterior leaflet. The maximum level of positioning is dictated by the ventricular muscle mass that impedes clamping further at the epicardial side. Nevertheless, the coronary sinus (CS) is compromised within the clamp. The posterolateral artery $(P L)$ of the right coronary can be seen in its epicardial path.

shown incomplete surgical lesions at the mitral and cavotricuspid isthmus. ${ }^{9}$ It is important to remark that atrial flutter is most of the time more symptomatic and worse tolerated than long-standing persistent AF, and if the narrow atrial tissue not successfully ablated lies beneath the Dacron annulus of a newly implanted valve prosthesis, the possibilities that postoperative flutter may be controlled in the electrophysiology laboratory are not precisely high.

Our observations in this study are founded in basic anatomy that we believe needs to be emphasized with the increase of procedures based on new technologies. The left atrial wall folds down internally over the crest of the left ventricle for a variable distance. On the epicardial side, the adi-

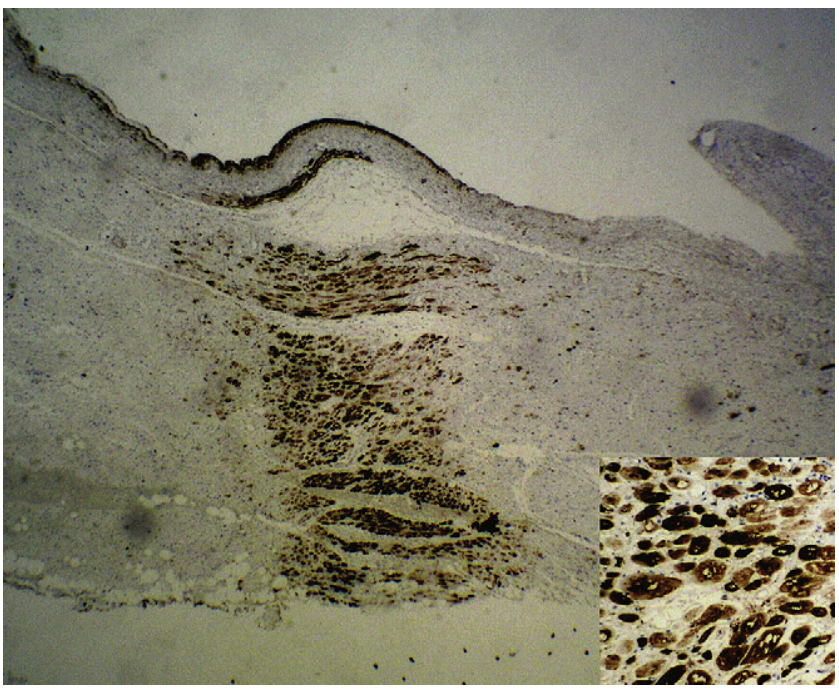

Figure 5. Complement C9 immunostaining of the posterior wall of the left atrium showing transmural necrosis approximately 8 hours after ablation was performed (original magnification $\times 20$ ). Inset, Zoomed view in the left inferior corner of a section of the same picture (original magnification $\times \mathbf{2 0 0}$ ).

and the structures that are embraced by this fat, the coronary arteries and sinus, lie in the epicardial side of both the left and right atria. This anatomic fact was already noted in the original descriptions by Cox of his technique, which included the use of a No. 15 blade knife into the epicardial fat through the overlapping atrial muscle to reach the annulus. This fat pad around the AV junction facilitates the progression in thickness from a thin atrial wall to a thick ventricular wall, most evident in the left side.

When applying the bipolar radiofrequency clamps in the left atrium to reach the mitral annulus, we detected two potential problems. First, the change in thickness from the atrium to the ventricle by the epicardial adipose tissue in the AV junction impeded closure of the clamp reaching the mitral annulus, leaving 5 to $10 \mathrm{~mm}$ of atrial muscle out of reach of the thermal
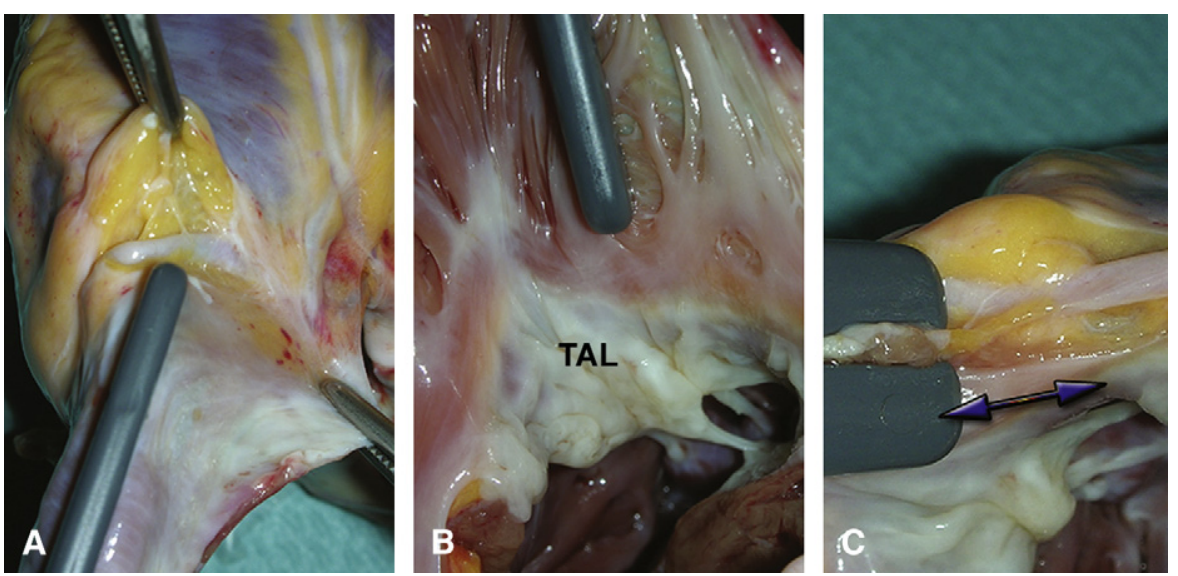

Figure 4 . The radiofrequency clamp is positioned to reach the tricuspid annulus. Limited by the right coronary artery in the epicardial side (A), the endocardial jaw of the clamp does not reach the annulus (B). In a side view (C) the arrows show the atrial myocardium from the electrodes to the annulus that is free from the thermal ablation. TAL, Tricuspid anterior leaflet. 
injury. This was confirmed in our postmortem ablation study, in which we could confirm normal myocardial tissue between the ablation injury and the mitral annulus. Second, even if the mitral annulus could not be reached, the coronary sinus and the circumflex artery in the left coronary-dominant hearts were included within the clamp, inasmuch as both structures lie in the atrial side of the AV junction.

In the right side, the slim thickness of both the atrial and free wall of the right ventricle allow closure of the clamp to reach the tricuspid annulus. However, then it always includes the midsection of the right coronary artery, inasmuch as this artery also lies in the atrial side of the AV junction. If this artery is to be avoided, the clamp is always unable to reach the tricuspid annulus.

This study was performed in freshly dead human hearts, and lesions were not actually performed. Therefore, we cannot conclude whether application of bipolar radiofrequency energy during a variable time between 5 to 15 seconds at around $60^{\circ} \mathrm{C}$ would irreversibly affect coronary circulation. Several studies report coronary damage by endocardially applied unipolar radiofrequency ablation, including temporal spasm by neurogenic or chemotactic (peptidergic) mechanisms. ${ }^{10}$ Therefore, and as intuitively advanced, patients with left coronary dominance may not be suitable candidates for bipolar radiofrequency or any means of epicardially applied thermal lesions to perform the mitral line. This can also be true in patients with important posterolateral branches arising from the right coronary artery. Careful revision of the preoperative coronary arteriogram is mandatory before deciding the ablation pattern in each patient.

Bipolar radiofrequency ablation toward the mitral annulus certainly produces a circumferential thermal ablation around the coronary sinus. To our knowledge, there are no reports of coronary sinus perforation or bleeding as a result of the application of bipolar clamps. Whether the thermal lesion creates a certain degree of stenosis is yet to be defined, as it has been the case in the pulmonary veins when percutaneous monopolar radiofrequency is endocardially applied with the circumferential Lasso catheter. Nevertheless, the multiple connections of the cardiac venous system with the ventricular cavities may limit the importance of possible coronary sinus stenosis.

In conclusion, current bipolar radiofrequency ablation technology is not able to complete a Cox maze IV procedure. A unipolar energy source, or ultimately the cut-and-sew technique, is needed to perform ablation lines reaching the mitral or the tricuspid valve annuli. At this time, only the cut-andsew technique ensures total transmurality along the lesion pattern of the Cox maze surgical procedure.

We thank the collaboration of the staff at the Transplant Services Foundation, especially Dr Elba Agustí. Likewise, we thank Ms Mar Pons for photographic assistance.

Disclosures and Freedom of Investigation. The bipolar clamps tested in the study were previously used in the clinical setting. Hearts were studied after homograft harvesting in our homograft bank (Transplant Services Foundation, Hospital Clínic, Barcelona, Spain) except one heart which was studied 18 hours after aortic valve replacement and radiofrequency Cox maze IV ablation. The study had no external funding. We had full control of the design of the study, methods used, outcome parameters, analysis of data, and production of the written report.

\section{References}

1. Cox JL, Boineau JP, Schuessler RB, Kater KM, Ferguson TB Jr, Cain ME, et al. Electrophysiologic basis, surgical development, and clinical results of the maze procedure for atrial flutter and atrial fibrillation. Adv Card Surg. 1995;6:1-67.

2. Haissaguerre M, Jais P, Shah DC, Takahashi A, Hocini M, Quiniou G, et al. Spontaneous initiation of atrial fibrillation by ectopic beats originating in the pulmonary veins. N Engl J Med. 1998;339:659-66.

3. Gillinov AM, Bhavani S, Blackstone EH, Rajeswaran J, Svensson LG, Navia JL, et al. Surgery for permanent atrial fibrillation: impact of patient factors and lesion set. Ann Thorac Surg. 2006;82:502-14.

4. Stulak JM, Dearani JA, Sundt TM 3rd, Daly RC, McGregor CG, Zehr KJ, et al. Superiority of cut-and-sew technique for the Cox maze procedure: comparison with radiofrequency ablation. J Thorac Cardiovasc Surg. 2007; 133:1022-7.

5. Piercecchi-Marti MD, Lepidi H, Leonetti G, Vire O, Cianfarani F, Pellissier JF, et al. Immunostaining by complement C9: a tool for early diagnosis of myocardial infarction and application in forensic medicine. J Forensic Sci. 2001;46:328-34.

6. Gaita F, Riccardi R, Caponi D, Shah D, Garberoglio L, Vivalda L, et al. Linear cryoablation of the left atrium versus pulmonary vein cryoisolation in patients with permanent atrial fibrillation and valvular heart disease: correlation of electroanatomic mapping and long-term clinical results. Circulation. 2005;111:136-42.

7. Calo L, Lamberti F, Loricchio ML, De Ruvo E, Colivicchi F, Bianconi L, et al. Left atrial ablation versus biatrial ablation for persistent and permanent atrial fibrillation: a prospective and randomized study. J Am Coll Cardiol. 2006;47:2504-12.

8. Perez FJ, Wood MA, Schubert CM. Effects of gap geometry on conduction through discontinuous radiofrequency lesions. Circulation. 2006; 113:1723-9.

9. Akar JG, Al Chekakie MO, Hai A, Brysiewicz N, Porter M, Varma N, et al. Surface electrocardiographic patterns and electrophysiologic characteristics of atrial flutter following modified radiofrequency MAZE procedures. J Cardiovasc Electrophysiol. 2007;18:349-55.

10. Simon RDB, Gill JS. Coronary ischemia induced by radiofrequency ablation in the left atrium. J Cardiovasc Electrophysiol. 2003;14:186-90. 